\title{
Utilizing Predictive Inflammatory Markers for Guiding the Use of Biologicals in Severe Asthma
}

\author{
Martin Runnstrom' \\ Hilary Pitner ${ }^{2}$ \\ Jennifer $\mathrm{Xu}^{2}$ \\ F Eun-Hyung Lee' \\ Merin Kuruvilla $\mathbb{D}^{1}$ \\ 'Division of Pulmonary, Allergy, Critical \\ Care and Sleep Medicine, Emory \\ University, Atlanta, GA, USA; \\ ${ }^{2}$ Department of Medicine, Emory \\ University, Atlanta, GA, USA
}

\begin{abstract}
Asthma is a chronic respiratory disease characterized by chronic airway inflammation and airflow obstruction. Up to ten percent of asthmatics have severe asthma, and many remain uncontrolled despite optimal medical management. With our increased understanding of the heterogeneity of asthma and its complex pathophysiology, several biomarkers have been developed and in the recent past, several biologic therapies for severe asthma have been developed and are now in widespread use. Although these biological agents have shown great benefit in treating severe asthma, not all patients respond equally well, and some do not derive any benefit. As much of the current literature of these medications have not assessed biomarkers or have used different cutoffs, it is often challenging to decide the best medication for an individual patient. Here, we review common asthma subtypes, current available biologic therapies for asthma, the clinical application of currently available type 2 biomarkers, as well as summarizing the evidence on how patient characteristics and biomarkers can help with choosing the optimal biologic for a patient that has the highest likelihood of success.
\end{abstract}

Keywords: asthma, biomarkers, biologics, eosinophils, immunoglobulin E, fractional exhaled nitric oxide

\section{Introduction}

Asthma is a chronic respiratory illness that is characterized by chronic airway inflammation and airflow obstruction. Approximately 5-10\% of asthmatic patients have severe asthma refractory to standard high-intensity therapies. ${ }^{1}$ Our evolving understanding of the heterogeneity of asthma and its complex pathophysiology has prompted the development of biomarkers to help guide treatment options especially for those with severe asthma. A biomarker is a measurable indicator of a pathological disease which can guide therapeutic options. Characteristics of a good biomarker include having a high predictive value of disease states, easy to obtain, widely available, affordable, and providing information about clinical outcome and prognosis.

Currently, biomarkers are frequently used in clinical settings to differentiate patients with severe asthma to determine optimal biologic treatment. Asthma is broadly divided into two categories: type 2 (T2-high) and non-type 2 (T2-low). ${ }^{2}$ In the era of personalized medicine, biomarkers provide insight into disease pathophysiology to guide biologic treatment selection to improve treatment success.

This review will describe the clinical application of biomarkers and how to select biologic therapies for type 2 asthma based upon asthma endotypes.
Correspondence: Merin Kuruvilla

Email merin.kuruvilla@emoryhealthcare.org 


\section{Asthma Phenotypes/Endotypes}

Asthma is an encompassing term that describes a heterogeneous group of diseases. The clinical features of asthma including shortness of breath, cough, and wheezing are driven by several different molecular pathways that cause dysregulated inflammatory responses. Traditionally asthma has been grouped by phenotypes, an observable set of characteristics which are a result of environmental and hereditary influences. However, as the biologic mechanisms of asthma have become more understood, our classification are shifting towards "molecular phenotypes" or endotypes. Asthma endotypes are subtypes differentiated by having distinct pathophysiological mechanisms. ${ }^{2}$ The shift towards using endotypes to classify asthma has created an opportunity for directed biologic therapies to make clinical impact.

There are two main asthma endotypes: T2-high and T2low. The T2-high endotype of asthma is driven by T2 cytokines (IL-4, IL-5, IL-13), eosinophils, alarmins (IL-25, IL-33, thymic stromal lymphopoietin [TSLP]), and Immunoglobulin $\mathrm{E}$ (IgE). Figure 1 outlines the type 2 inflammatory pathways in asthma. Two main groups of patients fall into this T2-high classification: early onset allergic asthma and late onset eosinophilic asthma. Early onset allergic asthma or atopic asthma affects mostly children and $\sim 50 \%$ of adults. ${ }^{3}$ It is the most common form of asthma and defined by high serum $\operatorname{IgE}$ or positive skin testing with comorbid conditions such as atopic dermatitis and allergic rhinitis. Late onset eosinophilic asthma affects mostly adults and characterized by high blood and sputum eosinophilia or Fractional Exhaled nitric oxide (FeNO) and is often dependent on systemic corticosteroid treatment. These patients also have a greater likelihood of having comorbid chronic rhinosinusitis and with nasal polyposis.

The mechanism of T2-low endotype is still poorly understood and to date there are no established biomarkers. ${ }^{4}$ T2-low asthma is characterized by neutrophilic or paucigranulocytic inflammation and likely involves the dysregulation of $\mathrm{T}$ helper (Th) cells 1, Th17 cells, and interferon gamma (IFN-y). ${ }^{2,4}$ Phenotypes associated with T2-low asthma include obesity, smoking, and very late onset (age $>50$ ) disease. ${ }^{2}$

Understanding the different phenotypes and endotypes of asthma helps delineate pathophysiology and can guide biologic

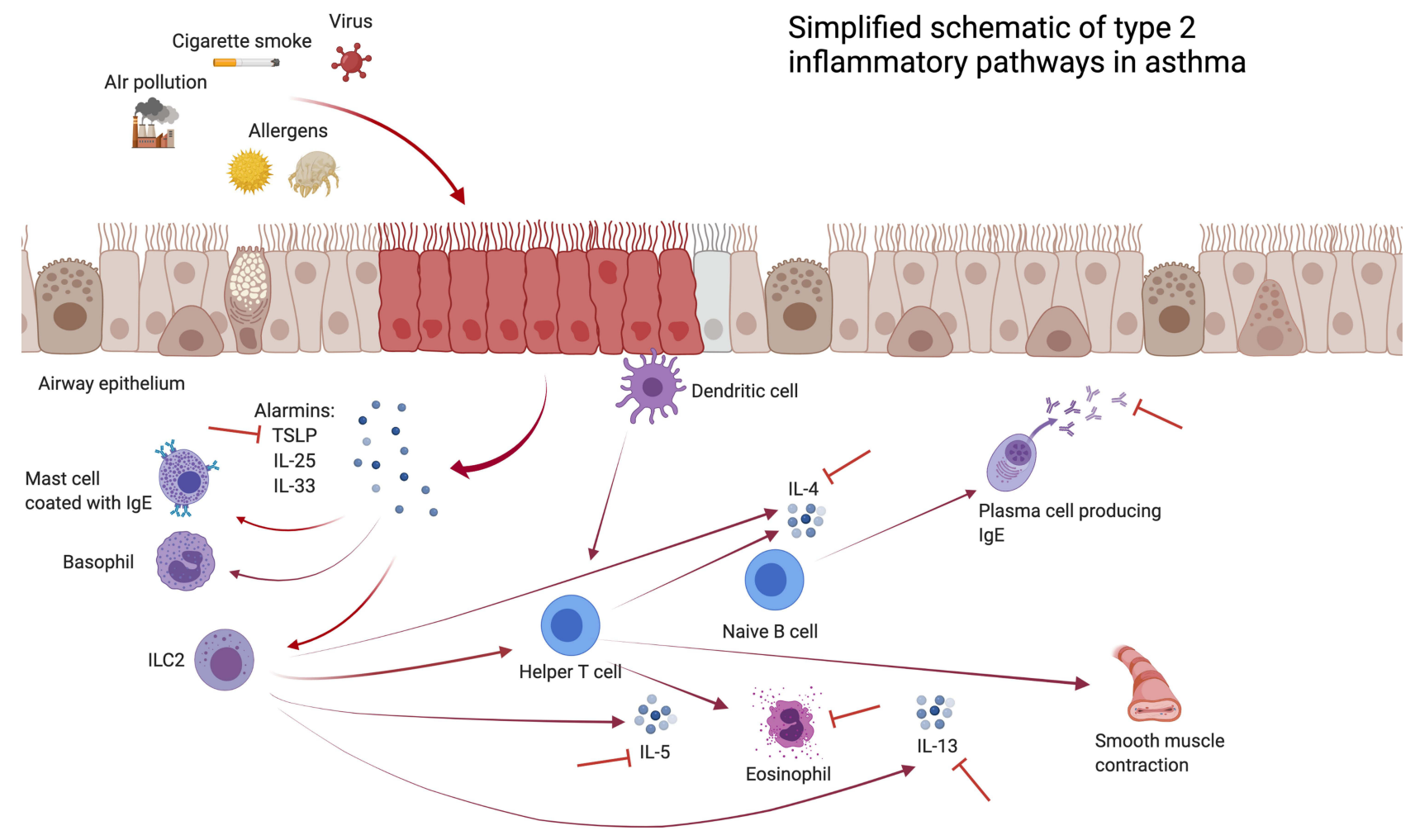

Figure I Simplified schematic of type 2 inflammatory pathways in asthma. Created with BioRender.com. 
therapy and patient outcomes. In this review, we will concentrate on the utility of biomarkers for type 2-high asthma as there are no validated T2-low asthma biomarkers in clinical practice.

\section{FDA Approved Biologic Treatments for Asthma}

There are multiple biologic therapies that target the T2high endotype, but none specifically directed at the T2-low endotype. At the time of writing this manuscript, there are six FDA approved biologics for the treatment of severe refractory asthma: omalizumab (anti-IgE), dupilumab (anti-IL4/IL13), three anti-eosinophil agents (mepolizumab, reslizumab, benralizumab), and anti-TSLP (tezepelumab).

Omalizumab was the first biologic therapy to be approved for asthma. It is a recombinant monoclonal antibody that binds to free IgE secreted in response to inhalants and interferes with allergic responses to environmental allergens. Omalizumab is approved to treat adults and children 6 years of age or older with moderate or severe persistent asthma not controlled with inhaled corticosteroids. Patients generally need to have a serum total IgE level between 30 and $700 \mathrm{IU} / \mathrm{mL}$ (IgE 30-1300 IU/ $\mathrm{mL}$ in 6-11 years of age) and evidence of in vitro perennial aeroallergen reactivity or positive skin test to qualify and lack other underlying conditions that could be the cause of the $\operatorname{IgE}$ elevation. $^{5}$

Mepolizumab is a humanized monoclonal antibody against IL-5 and the first approved anti-eosinophil agent. It was shown to decrease asthma exacerbations among severe eosinophilic asthmatics by $\sim 50 \%$. It is currently approved for severe asthmatics 6 years or older. Another FDA approved IL-5 antagonist is reslizumab. Its dosing is weight based, which has been hypothesized to potentially be advantageous in overweight patients if alternative medications would be relatively underdosed, although no head-to-head trials have evaluated this. Mepolizumab and reslizumab antagonize IL-5, the major signaling protein for eosinophil differentiation, proliferation, chemotaxis and survival. Benralizumab is an IL-5 receptor alpha antagonist that prevents IL-5 binding to its receptor and also elicits antibody-dependent cellular cytotoxicity against basophils and eosinophils. It is approved for severe asthmatics 12 years and above.

Dupilumab is a monoclonal antibody targeting the IL-4 receptor alpha, that serves as the common receptor for the IL-4 and IL-13 pathways and thus interferes with IL-4/IL-13 responses. Dupilumab is approved for ages 6 and above and is given as a subcutaneous injection every 2 weeks. To qualify, patients need to have uncontrolled eosinophilic asthma with elevated FeNO or elevated peripheral blood eosinophils (PBE) or be corticosteroid dependent. Finally, tezepelumab is the most recently approved biologic medication for severe asthma and targets TSLP, an upstream signaling molecule. It was shown to decrease the number of asthma exacerbations and improve FEV1 independent of biomarker status and is approved for adults and children at least 12 years of age without phenotype or biomarker limitations. ${ }^{58}$

\section{Biomarkers of Type 2 Inflammation in the Context of Type 2 Directed Biologics}

Biologic treatments for patients with severe asthma are not always successful and non-responsive patients need to discontinue therapy. The prediction of response to biologics has thus gained significant attention in the management of severe asthma. Currently, there are 4 commonly utilized biomarkers of T2-high asthma: sputum cell count, peripheral blood eosinophil count, serum immunoglobulin $\mathrm{E}(\mathrm{IgE})$ and fraction of exhaled nitric oxide (FeNO), ${ }^{6}$ that are the most valuable in understanding the underlying immunopathology driving asthma and for biologic selection. Although there is some degree of relationship between PBE count, FeNO, IgE and sputum eosinophilia (defined as $3 \%$ or higher), a systematic review found only moderate correlation. $^{7}$

Our understanding of biomarkers is limited by the fact that Phases 1 to 3 clinical trials for biologics are primarily focused on assessing efficacy and safety. Studies exploring expected responses to biologic therapy are based largely on post-hoc analyses of initial registration trials or small real-world studies. Regardless, clinical guidelines recommend obtaining multiple biomarkers in severe asthma, ie, blood eosinophils (threshold $>150$ cells $/ \mu \mathrm{L}$ ), FeNO (threshold $\geq 20 \mathrm{ppb}$ ), and $\mathrm{IgE}$ (threshold $\geq 30 \mathrm{IU} / \mathrm{mL}$ ), ${ }^{8}$ since only one biomarker may be increased independently from the others in some cases. Figure 2 presents a workflow for biologic therapy selection in severe asthma based on type 2 biomarkers.

\section{Blood Eosinophils}

Elevated blood eosinophils is the most established biomarker to predict the therapeutic efficacy of all currently available biologics for severe asthma except omalizumab. Eosinophilic inflammation is a downstream effect of various type- 2 cytokines. Since the routine measurement of 


\section{Algorithm for biologic therapy selection for severe asthma}

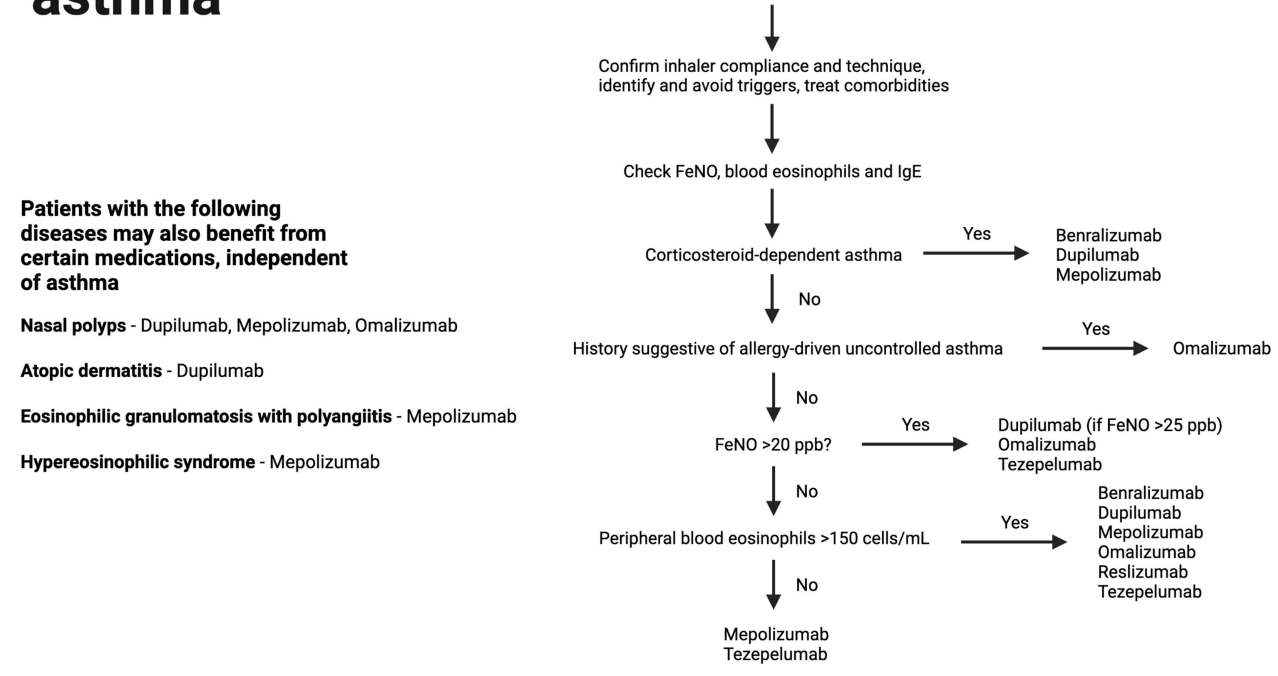

Uncontrolled asthma symptoms or exacerbations

Figure 2 Algorithm for biologic therapy selection for severe asthma. Created with BioRender.com.

cytokine levels is not feasible, PBE assessment is a more easily obtainable surrogate biomarker of type 2 inflammation. ${ }^{9}$ While PBE measurement has advantages of practicality, minimal invasiveness, and low cost, it is important to note that PBE counts are dynamic and fluctuate temporally. ${ }^{10}$ While longitudinal sampling could compensate for this aspect, repeated testing is demanding and limits applicability. Conversely, a previous analysis found that a single measurement of blood eosinophils was adequate to identify patients who might respond well to treatment, and multiple measurements barely increased sensitivity. ${ }^{11}$

All currently approved biologics for severe asthma demonstrated overall greater effectiveness in patients with higher baseline blood eosinophil levels, except for omalizumab. ${ }^{12,58}$ The degree of blood eosinophilia has been consistently identified as the primary baseline characteristic predictive of treatment response to the different anti-IL-5 agents in terms of decreased exacerbations and improvement in lung function. In general, a baseline PBE $<150$ cells $/ \mu \mathrm{L}$ precludes their use.

The development of mepolizumab highlighted the utility of blood eosinophils as a predictive biomarker of biologic responsiveness. Mepolizumab reduced rates of asthma exacerbations by almost half in severe eosinophilic asthma with a greater reduction in patients with higher eosinophils. ${ }^{13}$ This was reproduced in a secondary analysis of the DREAM and MENSA studies, in which mepolizumab showed greater clinical efficacy with higher baseline PBE as well as in patients with more frequent exacerbations. ${ }^{14}$ This post-hoc analysis showed that patients with PBE of at least 300 cells $/ \mu \mathrm{L}$ derived the greatest benefit. Overall, the higher the PBE, the greater the risk reduction with mepolizumab. ${ }^{15}$ For example, for $\mathrm{PBE}<150$ cells $/ \mu \mathrm{L}$ the rate ratio was 0.55 (95\% confidence interval (CI) $0.34-0.89$ ) for clinically significant exacerbation, as compared with patients with $\mathrm{PBE}>750$ cells $/ \mu \mathrm{L}$ for whom the rate ratio was 0.15 (95\% CI 0.08-0.29). ${ }^{15}$

Even though mepolizumab is currently approved for use in patients with PBE of at least 150 cells $/ \mu \mathrm{L}$, it is important to emphasize that a PBE threshold not be considered a static value as not only does it vary over time, but also may be decreased by things as corticosteroid use (eg, patients using oral corticosteroids might still have clinical benefit from these therapies despite a lower PBE). ${ }^{14}$

Similarly, studies of the other anti-eosinophil agents have confirmed the predictive nature of blood eosinophils. While treatment with reslizumab was associated with an overall reduction in blood eosinophil counts and improved lung function, this agent does not afford meaningful improvement of other asthma outcomes such as symptoms 
and lung function in patients with absolute eosinophil count (AEC) less than 400 cells $/ \mu \mathrm{L} .{ }^{16}$ The ZONDA trial showed that benralizumab led to significant reductions of oral corticosteroid use and asthma exacerbations (75\% and 70\% respectively) in patients with $\mathrm{PBE}$ of at least 150 cells $/ \mu \mathrm{L}$. Similar to the other anti-eosinophil agents, however, the most favorable responses to benralizumab were in patients with eosinophil counts of at least 300 cells $/ \mu \mathrm{L} .{ }^{17}$

In the phase 3 NAVIGATOR trial, tezepelumab decreased the number of asthma exacerbations and decreased blood eosinophils. In pre-specified subgroup analyses, the benefit was greater the higher the blood eosinophil count or FeNO, although a benefit was seen in all groups. ${ }^{58}$ Dupilumab treatment decreases asthma exacerbations and increases forced expiratory volume in the first second (FEV1) regardless of baseline PBE, but the benefits are greater the higher the baseline PBE. Among patients with $\mathrm{AEC} \geq 300$ cells $/ \mu \mathrm{L}$, dupilumab treatment decreased severe exacerbations by around two thirds relative to placebo in contrast with patients with AEC $<150$ cells/ $\mu \mathrm{L}$, where there was no difference. ${ }^{18,19}$

Finally, a post-hoc analysis of the EXTRA study of omalizumab showed greater treatment effect in the subgroup with an AEC of at least $260 / \mu \mathrm{L} .{ }^{20}$ However, two other studies reported discrepant results. In the STELLAIR study, omalizumab had equivalent effectiveness in both eosinophil "high" and "low" subgroups. ${ }^{21}$ Similarly, in the real-world PROSPERO study, improvements in omalizumab occurred independent of baseline eosinophils. ${ }^{22}$

It is intuitive to assume that the presence of significant eosinophilia despite systemic corticosteroids may herald even greater benefit from biologic medication. Rabe et al reported a consistent trend towards greater benefit with higher eosinophils compared to lower in corticosteroid dependent patients treated with dupilumab. ${ }^{18}$ In the study by Nair et al, however, among patients with severe asthma on corticosteroids, treatment with benralizumab reduced the dose of corticosteroid. ${ }^{23}$ However, in a minority of patients there was no reduction in corticosteroids, and those patients had similar eosinophil counts as responders. This indicates a different mechanism driving uncontrolled disease. This was also an exploratory outcome limited by low numbers. Similarly, in a cohort of 250 patients with at least 4 injections of reslizumab or mepolizumab, the use of daily prednisone and dose of prednisone were associated with increased risk of non-responsiveness. ${ }^{24}$

While these data overwhelmingly support the use of blood eosinophils in predicting response to biologic therapy, it is important to acknowledge the limitations. While an overall good indicator of type 2 inflammation, blood eosinophils do not always accurately reflect the presence and degree of airway inflammation. ${ }^{25,26}$ Hastie et al described a lack of correlation between blood and sputum eosinophils, the $\mathrm{R}$ value being 0.19 with $\sim 40 \%$ misclassification. ${ }^{27}$ Overall, PBE counts $<90$ cells $/ \mu \mathrm{L}$ are unlikely to correlate with sputum eosinophilia and conversely, PBE counts $>400$ cells/ $\mu \mathrm{L}$ are suggestive of significant airway eosinophils in the appropriate clinical context. $^{28,29}$ Additionally, eosinophils play a role in glucose homeostasis, so blood eosinophils might provide less accurate predictability of treatment response in obese patients. ${ }^{30}$ Finally, there can be variation across different laboratories and efforts should be made to standardize values. ${ }^{31}$

\section{Sputum Eosinophils}

The relationship between severe asthma and sputum eosinophilia has been long established. ${ }^{32}$ The demonstration of sputum eosinophils is considered the gold standard for the diagnosis of type 2 asthma. The use of sputum eosinophils as a predictive biomarker provides information about the state of the airway on a cellular level. A recent study of severe asthmatics demonstrated increased exacerbation frequency with increasing sputum eosinophilia. ${ }^{33}$ However, there are significant cost, availability and time barriers, preventing the widespread use of this biomarker.

Recurrent and persistent sputum eosinophilia $>3 \%$, but typically $>2 \%$, suggests eosinophils as the key effector cell in type 2 asthma. However, while sputum eosinophilia correlates with airway eosinophils, sputum eosinophil counts were not predictive of response to mepolizumab in the sputum sub-study of the DREAM trial. ${ }^{34}$ In this substudy of 94 patients, exacerbation rates were decreased by $\sim 70 \%$ regardless of sputum eosinophilia. This lack of a distinctive response based on sputum eosinophilia is counterintuitive because this biomarker likely represents the airway microenvironment better than PBE, and requires validation in further studies.

Measuring sputum eosinophils may also be useful to monitor response to therapies. The Phase 3 clinical trials for mepolizumab and reslizumab showed that treatment led to significant reductions in sputum eosinophils ${ }^{35,36}$ and persistent airway eosinophilia despite mepolizumab treatment indicates residual type 2 inflammation mediated by IL-5. ${ }^{24}$ In a cross-sectional study, standard monthly doses of mepolizumab $100 \mathrm{mg}$ subcutaneously 
did not suppress local airway eosinophilopoiesis, as defined by persistently elevated sputum eosinophil progenitor cells. Therefore, targeting IL-5-mediated local eosinophil differentiation within the airway may be relevant to optimal control in severe eosinophilic asthma. This was corroborated in a separate trial showing that suboptimal responders to mepolizumab with persistent sputum eosinophilia had improved clinical outcomes with decreased sputum eosinophils following switch to reslizumab. ${ }^{37}$ Hence, sputum eosinophil measurement may be used to assess airway inflammation in nonresponders to anti-eosinophil therapies.

A few studies have also utilized sputum cytometry to assess airway inflammation during exacerbations while on antieosinophil therapies. Despite treatment with mepolizumab or reslizumab, exacerbations were predominantly eosinophilic and may respond to systemic glucocorticoids. ${ }^{38}$ On the contrary, most exacerbations while on benralizumab are likely non-eosinophilic and neutrophilic due to intercurrent infection. The blood eosinophil count may be normal in these patients.

Aside from anti-eosinophil agents, the anti-IgE therapy omalizumab depletes lung eosinophils among patients with allergic asthma, which likely is due to decreased inflammatory cell recruitment following allergen exposure. ${ }^{39}$ Finally, dupilumab has not been demonstrated to decrease airway eosinophils, although the EXPEDITION study is currently ongoing and is evaluating the effect of dupilumab on inflammatory cells in the bronchial mucosa.

\section{FeNO}

Fractional excretion of exhaled nitric oxide (FeNO) is elevated in type 2 asthma secondary to airway epithelium expression of nitric oxide synthase induced by IL-13. An elevated FeNO is considered a surrogate of type 2 inflammation. Its measurement is accessible, noninvasive, and reproducible without significant variation. ${ }^{40}$ With omalizumab, a pre-treatment FeNO $\geq 24$ parts per billion (ppb) was predictive of a favorable response to treatment in the EXTRA study, ${ }^{20}$ in which reductions in exacerbations were greater in patients with high versus low FeNO (53\% versus $16 \%$, respectively). Additionally, an increase in FeNO at week 12 following interruption of long-term treatment with omalizumab may predict future exacerbations. ${ }^{41}$ It may be beneficial to restart omalizumab in those patients, but it is currently unknown if this would ameliorate this increased exacerbation risk.
Omalizumab treatment has also been shown to decrease FeNO by 4.24 ppb at 48 weeks, compared with placebo. ${ }^{5}$

In the phase 3 LIBERTY QUEST study, patients receiving dupilumab with a baseline FeNO of at least 25 ppb had a $\sim 50 \%$ decreased risk of exacerbation compared with placebo, but for patients with a FeNO $<25 \mathrm{ppb}$, there was no significant difference. ${ }^{19}$ Therefore, FeNO can be used as a biomarker to predict response to dupilumab and the Global Initiative for Asthma (GINA) currently recommends using a FeNO cutoff $\geq 25$ ppb to use omalizumab or dupilumab. Dupilumab use also reduces FeNO over time from baseline. ${ }^{19}$ Pre-treatment FeNO level was shown to predict response to tezepelumab, and the FeNO level was also shown to decrease with treatment compared to placebo. Similarly to blood eosinophils, the higher the baseline FeNO the greater the reduction was in annualized asthma exacerbations, although a benefit was still observed in patients with a baseline FeNO less than 25 ppb. ${ }^{58}$

Unlike other biologics, FeNO does not predict response to anti-eosinophil agents. The DREAM study found that FeNO readings were unaffected by mepolizumab treatment and FeNO assessment during treatment should therefore not be used as a marker of response. ${ }^{42}$ Also, the baseline FeNO level did not appear to predict response to anti-IL5 therapies, with equivalent effectiveness in populations with high and low FeNO levels. In concordance with the DREAM study, real-world studies have reproduced this lack of association between baseline FeNO and response to anti-IL5/IL5R agents and unchanged levels during treatment. ${ }^{43,44}$ In a recent study of more than 200 patients, the clinical efficacy of mepolizumab and benralizumab was independent of the baseline FeNO even at levels $\geq 75 \mathrm{ppb}^{43}$

\section{Serum $\lg \mathrm{E}$}

While the relationship between $\operatorname{IgE}$ and atopic asthma has long been established, patients with non-atopic asthma may also exhibit increased serum $\operatorname{IgE}(>150 \mathrm{IU} / \mathrm{mL})$, when compared with healthy controls. ${ }^{45}$ The allergen specificity of IgE in non-allergic type 2 asthmatics remains elusive, although Staphylococcus aureus enterotoxin-specific IgE and local IgE against dust mite have been implicated. ${ }^{46,47}$

While the selection of omalizumab for asthma is contingent upon a baseline serum IgE of 30 to $1300 \mathrm{IU} / \mathrm{mL}$ for ages $6-12$, and 30-700 IU $/ \mathrm{mL}$ for older patients, a study that combined 7 trials for a total of 4308 patients (2511 treated with omalizu$\mathrm{mab})$ found that the reduction in exacerbation risk was independent of $\operatorname{IgE}$ level (IgE level above or below median of 148 
$\mathrm{IU} / \mathrm{mL}){ }^{48}$ A study by Pillai et al found that in non-atopic asthmatics, omalizumab decreased mucosal IgE-positive cells on bronchial biopsies and improved lung function compared with placebo. This raises the question that local mucosal IgE rather than serum $\operatorname{IgE}$ is important for the clinical response to anti-IgE therapy in asthma. ${ }^{49}$ Monitoring IgE levels after treatment initiation is similarly not useful as a marker of responsiveness to omalizumab.

In the QUEST study of dupilumab, treatment was effective in patients with both allergic and non-allergic asthma including patients with allergic asthma and baseline serum $\mathrm{IgE}>700 \mathrm{IU} / \mathrm{mL} .^{50}$ This is relevant since omalizumab is currently not indicated for this patient subset in the United States. Consistent with its mechanism, dupilumab reduced total serum $\operatorname{IgE}$ in allergic and non-allergic asthmatics, and decreased allergen-specific $\operatorname{IgE}$ in those with allergic asthma. ${ }^{50}$

Akin to FeNO, serum IgE does not predict responsiveness to anti-IL5 agents. In a post-hoc analysis of the DREAM study, mepolizumab effectiveness was not associated with baseline IgE. ${ }^{51}$ This was also noted in post-hoc analyses of the phase 3 SIROCCO and studies with benralizumab, wherein maintenance treatment with benralizumab resulted in similar reductions in exacerbation rates when stratified according to high or low $\operatorname{IgE}$ (threshold $150 \mathrm{kU} / \mathrm{L}$ ). ${ }^{52}$

\section{Other Type 2 Biomarkers}

\section{Serum Periostin}

Periostin is an extracellular matrix protein secreted by bronchial epithelial cells in response to IL-4/IL-13 and is detectable in peripheral blood. Periostin has been implicated in the regulation of mucus production, subepithelial fibrosis, and airway remodeling in asthma.

The EXTRA study for omalizumab showed significantly larger reductions in exacerbation rates in patients with high versus low periostin: $30 \%$ versus $3 \%$, respectively. $^{20}$ Tajiri et al later confirmed the utility of baseline serum periostin as a biomarker in predicting omalizumab response. ${ }^{53}$ In a cohort of 30 patients treated for at least 1 year, baseline serum periostin levels negatively associated with levels of free serum IgE after 16 to 32 weeks of therapy. Overall, the decrease in periostin levels correlated positively with reduced exacerbations over the two-year study period. Dupilumab also induced a rapid and sustained reduction in serum periostin in severe asthma as compared with placebo. ${ }^{54}$

There is limited data on the application of periostin measurement in the setting of treatment with anti-IL5
/IL5R agents, outside of a small study showing a significant decrease in nasal periostin levels with mepolizumab for the treatment of nasal polyps.

It must also be noted that periostin is not a straightforward surrogate marker of type 2 inflammation. Periostin is specifically linked with tissue remodeling in asthma, and does not necessarily correlate with eosinophils and other biomarkers. Therefore, the level of interest as a predictor of responsiveness to biologic treatments has waned in recent years. ${ }^{55}$

\section{Composite Biomarkers}

In addition to individual biomarkers, some studies have combined markers to enhance their predictive ability. A recent systematic review demonstrated augmented diagnostic accuracy for the combination of the AEC, serum IgE and FeNO for identifying sputum eosinophils. ${ }^{7}$ A prediction model in two separate cohorts was able to predict sputum eosinophils with $>70 \%$ sensitivity and specificity based on clinical phenotype combined with FeNO, PBE, and activation status of blood neutrophils and eosinophils. Another study showed that a composite score of three biomarkers (FeNO, PBE, and serum periostin) is an independent predictor of exacerbation risk in patients with severe asthma and this may support the decision to pursue biologic therapy. ${ }^{56}$ Some clinical trials have applied targeted biologic treatments based on patient profiles of composite biomarkers. ${ }^{57}$ The predictive ability of composite biomarker testing in biologic treatments for asthma needs to be established.

\section{Conclusion}

Although type 2 biological agents have been promising in severe asthma, not all patients respond equally well to these therapies. These differences in treatment response are likely multifactorial, due to the heterogeneity of underlying inflammatory pathways in severe asthma as well as drug-related factors. The optimal use of biologics is of utmost importance in terms of cost and prevention of needless exposure. The decision to initiate and maintain biological therapy is therefore contingent upon the recognition of the clinical relevance of biomarkers and phenotypes. Unfortunately, the selection of a biologic agent is not straightforward and predictive markers that herald responsiveness has gained recent attention.

Studies that have investigated predictive markers for biologic use have provided limited insight, but further evidence is strongly needed. Individual biomarkers are also not necessarily specific enough to allow for targeted treatment in 
type 2 asthma. For instance, PBE measurement predicts responsiveness to biologics targeting IL-4/IL-13, TSLP, IL5 or the IL-5 receptor, without clearly indicating which of these is best suited for an individual patient. A composite approach that incorporates clinical phenotypes, treatable traits, comorbidities, such as atopic dermatitis or nasal polyps, and inflammatory biomarkers may better address this question. At present, decisions on selection and maintenance of biologics for severe asthma are challenging and further research should evaluate real-world data and the utility of algorithms that leverage baseline and posttreatment parameters to predict long-term benefit.

\section{Abbreviations}

T2, type 2; IgE, Immunoglobulin E; FeNO, Fractional Exhaled nitric oxide; Th, T helper; PBE, peripheral blood eosinophils.

\section{Disclosure}

The authors report no conflicts of interest in this work.

\section{References}

1. Hekking P-PW, Wener RR, Amelink M, et al. The prevalence of severe refractory asthma. J Allergy Clin Immunol. 2015;135 (4):896-902. doi:10.1016/j.jaci.2014.08.042

2. Kuruvilla ME, Lee FE, Lee GB. Understanding asthma phenotypes, endotypes, and mechanisms of disease. Clin Rev Allergy Immunol. 2019;56(2):219-233. doi:10.1007/s12016-018-8712-1

3. Akar-Ghibril N, Casale T, Custovic A, et al. Allergic endotypes and phenotypes of asthma. J Allergy Clin Immunol Pract. 2020;8 (2):429-440. doi:10.1016/j.jaip.2019.11.008

4. Fitzpatrick AM, Chipps BE, Holguin F, et al. T2-"Low" asthma: overview and management strategies. J Allergy Clin Immunol Pract. 2020;8(2):452-463. doi:10.1016/j.jaip.2019.11.006

5. Hanania NA, Alpan O, Hamilos DL, et al. Omalizumab in severe allergic asthma inadequately controlled with standard therapy: a randomized trial. Ann Intern Med. 2011;154(9):573-582. doi:10.7326/0003-4819-154-9-201105030-00002

6. Lim H, Nair P. Airway inflammation and inflammatory biomarkers. Semin Respir Crit Care Med. 2018;39(01):056-063. doi:10.1055/ s-0037-1606217

7. Korevaar DA, Westerhof GA, Wang J, et al. Diagnostic accuracy of minimally invasive markers for detection of airway eosinophilia in asthma: a systematic review and meta-analysis. Lancet Respir Med. 2015;3(4):290-300. doi:10.1016/S2213-2600(15)00050-8

8. Boulet LP, Reddel HK, Bateman E, et al. The Global Initiative for Asthma (GINA): 25 years later. Eur Respir J. 2019;54(2):1900598. doi:10.1183/13993003.00598-2019

9. Peters MC, Wenzel SE. Intersection of biology and therapeutics: type 2 targeted therapeutics for adult asthma. Lancet. 2020;395 (10221):371-383. doi:10.1016/S0140-6736(19)33005-3

10. Corren J, Du E, Gubbi A, et al. Variability in blood eosinophil counts in patients with eosinophilic asthma. J Allergy Clin Immunol Pract. 2021;9(3):1224-1231 e9. doi:10.1016/j.jaip.2020.10.033
11. Ortega H, Gleich G, Mayer B, et al. Reproducibility of a single blood eosinophil measurement as a biomarker in severe eosinophilic asthma. Ann Am Thorac Soc. 2015;12(12):1896-1897. doi:10.1513/ AnnalsATS.201507-443LE

12. McGregor MC, Krings JG, Nair P, et al. Role of biologics in asthma. Am J Respir Crit Care Med. 2019;199(4):433-445. doi:10.1164/ rccm.201810-1944CI

13. Ortega HG, Liu MC, Pavord ID, et al. Mepolizumab treatment in patients with severe eosinophilic asthma. N Engl J Med. 2014;371 (13):1198-1207. doi:10.1056/NEJMoa1403290

14. Ortega HG, Yancey SW, Mayer B, et al. Severe eosinophilic asthma treated with mepolizumab stratified by baseline eosinophil thresholds: a secondary analysis of the DREAM and MENSA studies. Lancet Respir Med. 2016;4(7):549-556. doi:10.1016/S2213-2600(16)30031-5

15. Albers FC, Licskai C, Chanez P, et al. Baseline blood eosinophil count as a predictor of treatment response to the licensed dose of mepolizumab in severe eosinophilic asthma. Respir Med. 2019;159:105806. doi:10.1016/j.rmed.2019.105806

16. Corren J, Weinstein S, Janka L, et al. Phase 3 study of reslizumab in patients with poorly controlled asthma: effects across a broad range of eosinophil counts. Chest. 2016;150(4):799-810. doi:10.1016/j. chest.2016.03.018

17. Bleecker ER, Wechsler ME, FitzGerald JM, et al. Baseline patient factors impact on the clinical efficacy of benralizumab for severe asthma. Eur Respir J. 2018;52(4):1800936. doi:10.1183/13993003.00936-2018

18. Rabe KF, Nair P, Brusselle G, et al. Efficacy and safety of dupilumab in glucocorticoid-dependent severe asthma. N Engl J Med. 2018;378 (26):2475-2485. doi:10.1056/NEJMoa1804093

19. Castro M, Corren J, Pavord ID, et al. Dupilumab efficacy and safety in moderate-to-severe uncontrolled asthma. $N$ Engl J Med. 2018;378 (26):2486-2496. doi:10.1056/NEJMoa1804092

20. Hanania NA, Wenzel S, Rosén K, et al. Exploring the effects of omalizumab in allergic asthma: an analysis of biomarkers in the EXTRA study. Am J Respir Crit Care Med. 2013;187(8):804-811. doi:10.1164/rccm.201208-1414OC

21. Humbert M, Taillé C, Mala L, et al. Omalizumab effectiveness in patients with severe allergic asthma according to blood eosinophil count: the STELLAIR study. Eur Respir J. 2018;51(5):1702523. doi:10.1183/13993003.02523-2017

22. Casale TB, Luskin AT, Busse W, et al. Omalizumab effectiveness by biomarker status in patients with asthma: evidence from PROSPERO, A prospective real-world study. J Allergy Clin Immunol Pract. 2019;7 (1):156-164 e1. doi:10.1016/j.jaip.2018.04.043

23. Nair P, Wenzel S, Rabe KF, et al. Oral glucocorticoid-sparing effect of benralizumab in severe asthma. $N$ Engl J Med. 2017;376 (25):2448-2458. doi:10.1056/NEJMoa1703501

24. Mukherjee M, Forero, DF, Tran S, et al. Suboptimal treatment response to anti-IL-5 monoclonal antibodies in severe eosinophilic asthmatics with airway autoimmune phenomena. Eur Respir J. 2020;56(4):2000117. doi:10.1183/13993003.00117-2020

25. Price DB, Rigazio A, Campbell JD, et al. Blood eosinophil count and prospective annual asthma disease burden: a UK cohort study. Lancet Respir Med. 2015;3(11):849-858. doi:10.1016/S2213-2600(15)00367-7

26. Busse WW. Biological treatments for severe asthma: a major advance in asthma care. Allergol Int. 2019;68(2):158-166. doi:10.1016/j. alit.2019.01.004

27. Hastie AT, Moore WC, Li H, et al. Biomarker surrogates do not accurately predict sputum eosinophil and neutrophil percentages in asthmatic subjects. J Allergy Clin Immunol. 2013;132(1):72-80. doi:10.1016/j.jaci.2013.03.044

28. Wagener AH, de Nijs SB, Lutter R, et al. External validation of blood eosinophils, $\mathrm{FE}(\mathrm{NO})$ and serum periostin as surrogates for sputum eosinophils in asthma. Thorax. 2015;70(2):115-120. doi:10.1136/ thoraxjn1-2014-205634 
29. Zhang X-Y, Simpson JL, Powell H, et al. Full blood count parameters for the detection of asthma inflammatory phenotypes. Clin Exp Allergy. 2014;44(9):1137-1145. doi:10.1111/cea.12345

30. Lugogo N, Green CL, Agada N, et al. Obesity's effect on asthma extends to diagnostic criteria. J Allergy Clin Immunol. 2018;141 (3):1096-1104. doi:10.1016/j.jaci.2017.04.047

31. Fahy JV. Type 2 inflammation in asthma-present in most, absent in many. Nat Rev Immunol. 2015;15(1):57-65. doi:10.1038/nri3786

32. Nelson RK, Bush A, Stokes J, et al. Eosinophilic Asthma. J Allergy Clin Immunol Pract. 2020;8(2):465-473. doi:10.1016/j.jaip.2019.11.024

33. Denlinger LC, Phillips BR, Ramratnam S, et al. Inflammatory and Comorbid Features of Patients with Severe Asthma and Frequent Exacerbations. Am J Respir Crit Care Med. 2017;195(3):302-313. doi:10.1164/rccm.201602-04190C

34. Katz LE, Gleich GJ, Hartley BF, et al. Blood eosinophil count is a useful biomarker to identify patients with severe eosinophilic asthma. Ann Am Thorac Soc. 2014;11(4):531-536. doi:10.1513/ AnnalsATS.201310-354OC

35. Nair P, Pizzichini MMM, Kjarsgaard M, et al. Mepolizumab for prednisone-dependent asthma with sputum eosinophilia. $N$ Engl J Med. 2009;360(10):985-993. doi:10.1056/NEJMoa0805435

36. Haldar P, Brightling CE, Hargadon B, et al. Mepolizumab and exacerbations of refractory eosinophilic asthma. $N$ Engl J Med. 2009;360(10):973-984. doi:10.1056/NEJMoa0808991

37. Mukherjee M, Aleman Paramo F, Kjarsgaard M, et al. Weight-adjusted Intravenous Reslizumab in Severe Asthma with Inadequate Response to Fixed-Dose Subcutaneous Mepolizumab. Am J Respir Crit Care Med. 2018;197(1):38-46. doi:10.1164/rccm.201707-1323OC

38. Bhalla A, Zhao N, Rivas DD, et al. Exacerbations of Severe Asthma While on Anti-IL-5 Biologics. J Investig Allergol Clin Immunol. 2020;30(5):307-316. doi:10.18176/jiaci.0628

39. Djukanovic R, Wilson SJ, Kraft M, et al. Effects of treatment with anti-immunoglobulin $\mathrm{E}$ antibody omalizumab on airway inflammation in allergic asthma. Am J Respir Crit Care Med. 2004;170 (6):583-593. doi:10.1164/rccm.200312-1651OC

40. Kharitonov SA, Gonio F, Kelly C, et al. Reproducibility of exhaled nitric oxide measurements in healthy and asthmatic adults and children. Eur Respir J. 2003;21(3):433-438. doi:10.1183/09031936.03.00066903a

41. Ledford D, Busse W, Trzaskoma B, et al. A randomized multicenter study evaluating Xolair persistence of response after long-term therapy. $J$ Allergy Clin Immunol. 2017;140(1):162-169 e2. doi:10.1016/j. jaci.2016.08.054

42. Pavord ID, Korn S, Howarth P, et al. Mepolizumab for severe eosinophilic asthma (DREAM): a multicentre, double-blind, placebo-controlled trial. Lancet. 2012;380(9842):651-659. doi:10.1016/S0140-6736(12)60988-X

43. Hearn AP, Kavanagh J, d'Ancona G, et al. The relationship between Feno and effectiveness of mepolizumab and benralizumab in severe eosinophilic asthma. J Allergy Clin Immunol Pract. 2021;9(5):20932096 e1. doi:10.1016/j.jaip.2021.01.008

44. Ramonell RP, Lee FE-H, Levy JM, et al. Exhaled nitric oxide measurements are not influenced by anti-eosinophil therapy in patients with asthma: a retrospective analysis. Ann Allergy Asthma Immunol. 2021;126(1):102-104. doi:10.1016/j.anai.2020.09.005
45. Beeh KM, Ksoll M, Buhl R. Elevation of total serum immunoglobulin $\mathrm{E}$ is associated with asthma in nonallergic individuals. Eur Respir J. 2000;16(4):609-614. doi:10.1034/j.1399-3003.2000.16d07.x

46. Bachert C, Humbert M, Hanania NA, et al. Staphylococcus aureus and its IgE-inducing enterotoxins in asthma: current knowledge. Eur Respir J. 2020;55(4):1901592. doi:10.1183/13993003.01592-2019

47. Mouthuy J, Detry B, Sohy C, et al. Presence in sputum of functional dust mite-specific IgE antibodies in intrinsic asthma. Am J Respir Crit Care Med. 2011;184(2):206-214. doi:10.1164/rccm.20100914340C

48. Bousquet J, Cabrera P, Berkman N, et al. The effect of treatment with omalizumab, an anti-IgE antibody, on asthma exacerbations and emergency medical visits in patients with severe persistent asthma. Allergy. 2005;60(3):302-308. doi:10.1111/j.1398-9995.2004.00770.x

49. Pillai P, Chan Y-C, Wu S-Y, et al. Omalizumab reduces bronchial mucosal IgE and improves lung function in non-atopic asthma. Eur Respir J. 2016;48(6):1593-1601. doi:10.1183/13993003.01501-2015

50. Corren J, Castro M, O'Riordan T, et al. Dupilumab Efficacy in Patients with Uncontrolled, Moderate-to-Severe Allergic Asthma. J Allergy Clin Immunol Pract. 2020;8(2):516-526. doi:10.1016/j. jaip.2019.08.050

51. Ortega H, Chupp G, Bardin P, et al. The role of mepolizumab in atopic and nonatopic severe asthma with persistent eosinophilia. Eur Respir J. 2014;44(1):239-241. doi:10.1183/09031936.00220413

52. Chipps BE, Newbold P, Hirsch I, Trudo F, Goldman M. Benralizumab efficacy by atopy status and serum immunoglobulin E for patients with severe, uncontrolled asthma. Ann Allergy Asthma Immunol. 2018;120(5):504-511 e4. doi:10.1016/j.anai.2018.01.030

53. Tajiri T, Matsumoto H, Gon Y, et al. Utility of serum periostin and free $\operatorname{IgE}$ levels in evaluating responsiveness to omalizumab in patients with severe asthma. Allergy. 2016;71(10):1472-1479. doi:10.1111/all.12922

54. Hamilton JD, Harel S, Swanson BN, et al. Dupilumab suppresses type 2 inflammatory biomarkers across multiple atopic, allergic diseases. Clin Exp Allergy. 2021;51(7):915-931. doi:10.1111/ cea. 13954

55. Izuhara K, Ohta S, Ono J. Using periostin as a biomarker in the treatment of asthma. Allergy Asthma Immunol Res. 2016;8 (6):491-498. doi:10.4168/aair.2016.8.6.491

56. Heaney LG, Djukanovic R, Woodcock A, et al. Research in progress: medical Research Council United Kingdom Refractory Asthma Stratification Programme (RASP-UK). Thorax. 2016;71(2):187-189. doi:10.1136/thoraxjnl-2015-207326

57. Pettipher R, Hunter MG, Perkins CM, et al. Heightened response of eosinophilic asthmatic patients to the CRTH2 antagonist OC000459. Allergy. 2014;69(9):1223-1232. doi:10.1111/all.12451

58. Menzies-Gow A, Corren J, Bourdin A, et al. Tezepelumab in Adults and Adolescents with Severe, Uncontrolled Asthma. $N$ Engl J Med. 2021;13;384(19):1800-1809.
Journal of Inflammation Research

\section{Publish your work in this journal}

The Journal of Inflammation Research is an international, peerreviewed open-access journal that welcomes laboratory and clinical findings on the molecular basis, cell biology and pharmacology of inflammation including original research, reviews, symposium reports, hypothesis formation and commentaries on: acute/chronic inflammation; mediators of inflammation; cellular processes; molecular mechanisms; pharmacology and novel anti-inflammatory drugs; clinical conditions involving inflammation. The manuscript management system is completely online and includes a very quick and fair peerreview system. Visit http://www.dovepress.com/testimonials.php to read real quotes from published authors. 